www.jmscr.igmpublication.org

Impact Factor 3.79

Index Copernicus Value: 5.88

ISSN (e)-2347-176x ISSN (p) 2455-0450

crossref DOI:_http://dx.doi.org/10.18535/jmscr/v4i1.14

Journal Of Medical Science And Clinical Research

\title{
A Retrospective Study of Fungal Keratitis in VIMS, Bellary
}

\section{Authors \\ K. Ram Mohan Reddy, Sangeetha C Patil, Shafiyabi S, Krishna S}

\begin{abstract}
Introduction: Corneal infection is leading cause of ocular morbidity and blindness worldwide. To minimize ocular morbidity timely antifungal treatment must be initiated on the basis of clinical and microbiological evaluation. Culture and direct microscopic detection of causative fungi are the two important mycological investigations that are widely used.

Objective: To identify the causative fungi of infectious keratitis in patients attending ophthalmology department in VIMS, Bellary.

Materials and methods: A retrospective review of mycological records was conducted for all patients with infectious keratitis who presented between 01.04.2013 to 30.07.2013 at VIMS, Bellary. The scraping material obtained from leading edge and base of each ulcer was initially directly inoculated on to the surface of Sabauraud's dextrose agar in a row of ' $C$ ' shaped streak. The material obtained by the next scrapings was spread on to labeled slides in a thin; even manner for $10 \% \mathrm{KOH}$ wet mount and Gram's staining. The fungal isolates were identified using standard microbiological procedures.

Results: A total of 86 patients suspected of fungal keratits presented during the study period. 21 (24.42\%) of patients were culture positive. The most common fungal pathogen was Aspergillus spp (11) $52.4 \%$ of all positive fungal cultures, followed by Fusarium spp ${ }^{(6)} 28.6 \%$, of patients, and Mucor spp ${ }^{(2)} 9.5 \%$. Conclusion: In the present study Aspergillus spp was the most common fungus isolated. Prompt diagnosis and early institution of appropriate antifungal may limit the ocular morbidity and sequel of infectious keratitis. These regional findings have important public health implications for the treatment of corneal ulcerations in this part of India.
\end{abstract}

\section{Introduction}

Eye is one of the important organs of sensory perception. Normally it is kept free from microbial infections by the cleansing effect of lacrimal secretions. However, certain conditions like trauma to the eye ball and therapy with antibiotics and corticosteroids render the eye susceptible to infection with various fungal members with or without bacterial associates ${ }^{1}$. A large number of fungi have been incriminated to be the cause of mycotic keratitis. However, the frequency and spectrum of fungal members involved differs from place to place largely based on climatic, geographical and socio-economic conditions $^{2,3}$. Present study was done to identify the causative fungi of infectious keratitis in patients attending ophthalmology department in VIMS, Bellary.

\section{Materials and Methods}

After getting approval from the ethical committee, a retrospective review of mycological records was conducted for all patients with infectious keratitis 
who presented between 01.04.2013 and 30.07.2013 to Vijanagar Institue of Medical Sciences, Bellary, Karnataka, India ( VIMS). A total of 86 consecutive patients with corneal ulceration were analyzed. Ulceration was defined as a loss of the corneal epithelium with underlying stromal infiltration and suppuration associated with signs of inflammation with or without hypopyon. Corneal scrapings were collected from the edge of the base of the ulcers using fresh sterile Baird-Parker blades after anaesthetizing the eye with 2-3 drops of sterile local anesthetic solution. A direct smear prepared from a portion of the scrapings was stained by Gram's Method and examined microscopically both for fungal elements and bacteria. Scrapings were also examined in $10 \% \mathrm{KOH}$ wet preparations for fungal elements. The other portions of corneal scrapings were cultured both for fungi and bacteria. Duplicate Sabouraud's dextrose agar slants were inoculated with corneal scrapings to isolate fungi. One of the tubes was incubated at room temperature and the other at $37^{\circ} \mathrm{C}$. Both the tubes were examined daily for fungal growth. Positive cultures were identified by colonial morphology like texture, colour and by microscopic morphology in slide cultures. Fungal aetiology of corneal ulcers was considered based on either positively both in smear and culture or repeated appearance in smear or culture or in both. A routine culture for bacteria too was done: positive cultures identified and their antibiotic sensitivity pattern determined.

\section{Results}

\section{Microbiological Profile}

Fungi alone was isolated from 21patients $(24.42 \%)$, both bacteria and fungi from 2 patients (2.3\%) There was no growth in culture from the corneal scrapings of $61(69.3 \%)$ patients. The predominant fungal species isolated were. A total of 86 patients suspected of fungal keratits presented during the study period. 21 (24.42\%) of patients were culture positive. The most common fungal pathogen was Aspergillus spp (11) representing $52.4 \%$ of all positive fungal cultures, followed by Fusarium spp ${ }^{(6)}$ representing $28.6 \%$, of patients, and Mucor spp (2) $9.5 \%$. Other fungal species isolated were Cladosporium $1(4.8 \%)$ and Geotrichum 1 (4.8\%). There were 2 mixed infections (both bacterial and fungal) and in both the cases the bacteria associated was Staphylococcus aureus, and both the isolates of Staphylococcus aureus were sensitive to majority of first line antibiotics.

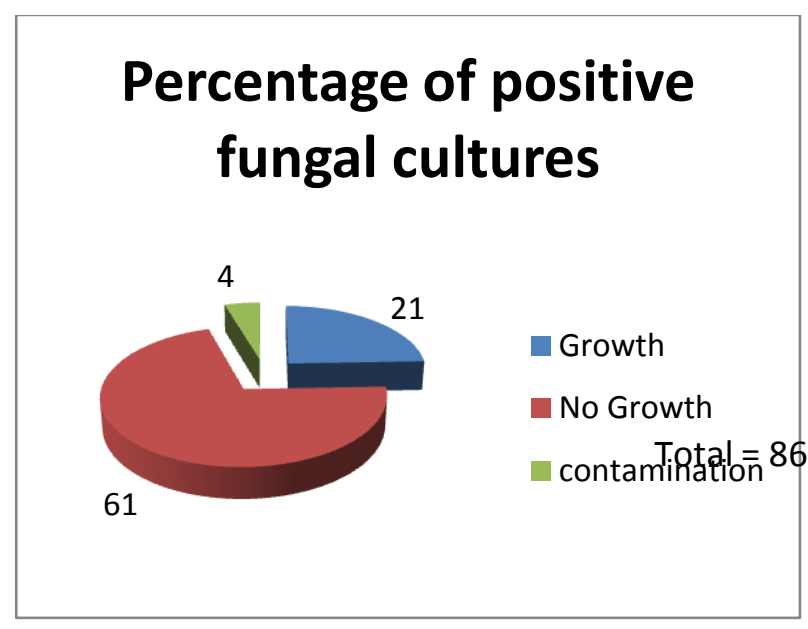

\section{Fungus isolated}

Aspergillus spp

Fusarium spp

Mucor spp

Cladosporiu

$\mathrm{m}$

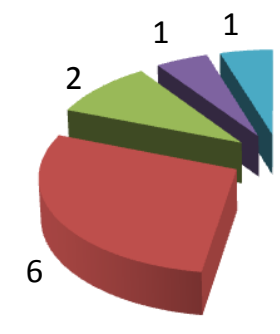

11

Geotrichum

\section{Demographics}

Out of 86 patients $30(34.9 \%)$ were females and $56(65.1 \%)$ were males. The age of the patients ranged from 8 to 71 years. Majority were in the ages groups of $21-30(17,19.78 \%)$ and $31-40$ $(17,19.78 \%)$ age groups. Males accounted for 
majority of the fungal growth $(15,71.43 \%)$, females contributed to $6,28.57 \%$.
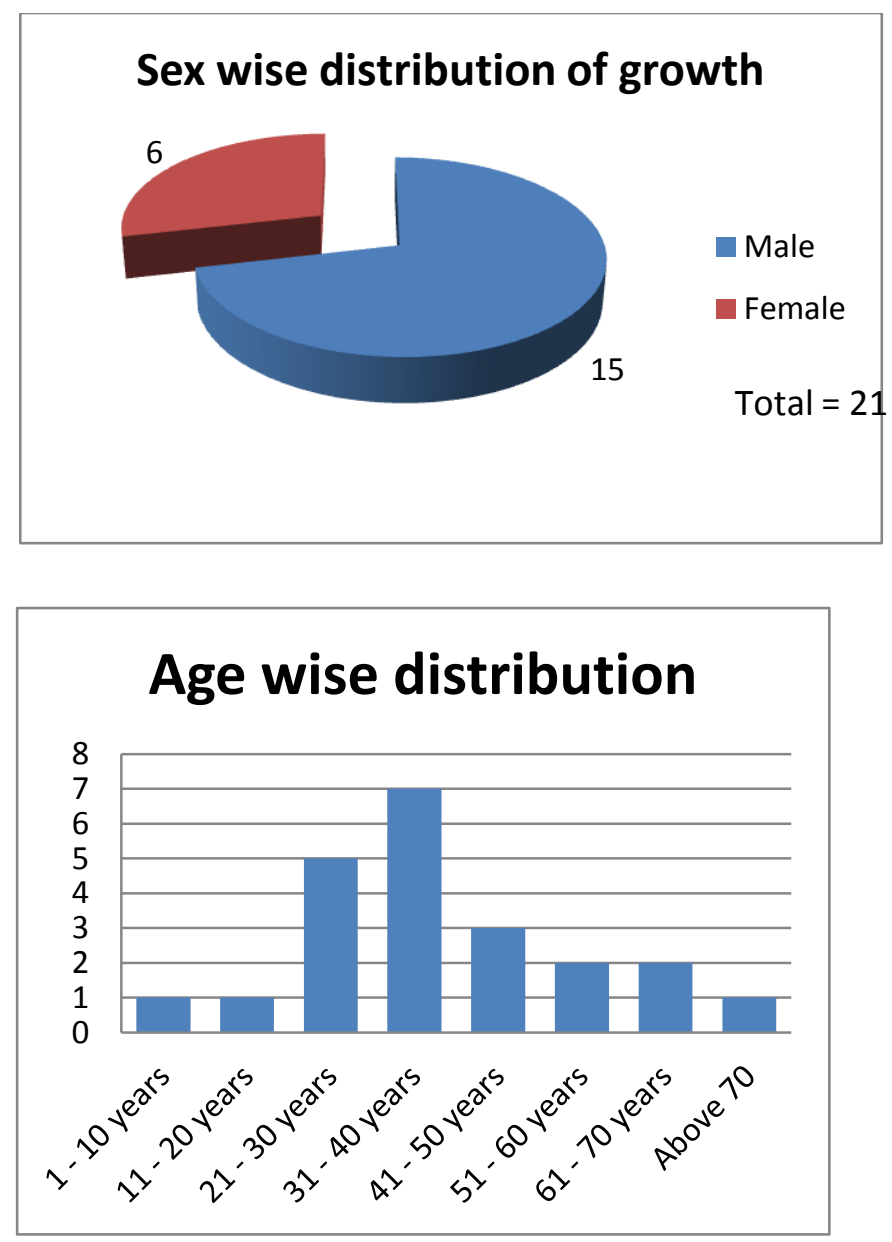

\section{Discussion}

In the present study Aspergillus spp was the most common fungus isolated, similar to other studies done $e^{4,5}$. As shown in the earlier studies done in different parts of south India, even in this study the incidence of fungal keratits was more common in the male age group, and in the age groups of 31 - 40 years, which represent the working age group $^{6,7} .62(70.9 \%)$ of the samples sent did not yield growth, indicating inappropriate sample collection as the most plausible explanation, bringing to need proper training of the ophthalmologist to collect a representative sample. Majority of patients attending to this hospital are from the rural areas, where the chief occupation is farming, trauma is the result of farming practices. In this study there were cases of fungal keratits in the school going age group, which could be attributed to the summer vacation during the months of April and May, during which children are at an increased risk of sport injuries. Prompt diagnosis and early institution of appropriate antifungal may limit the ocular morbidity and sequel of infectious keratitis. These regional findings have important public health implications for the treatment of corneal ulcerations in this part of India.

\section{Conclusion}

This study was done between the months of April and July, which is non-harvesting season. Though studies have shown no season wise distribution of fungal keratitis, the frequency and spectrum of fungal members involved differs from place to place largely based on climatic, geographical and socio-economic conditions. A more robust prospective study, is needed to look for distribution of fungal keratitis during harvesting season.

\section{References}

1. Norman. FC., David. T.S. et al. Manual of clinical mycology W.B.Saunders Company. Philadelphia. 3rd edition. 1971.

2. Subbannayya Kotigadde, Mamatha Ballal, Jyothirlatha, et al. "Mycotic keratitis: A study in coastal Karnataka". Indian Journal of Ophthalmology, (1992) 40 (1): 31-33.

3. MJ Bharathi, R Ramakrishnan, S Vasu, et al. "Epidemiology of Bacterial Keratitis in a referral centre in South India". Indian Journal of Medical Microbiology, (2003) 21 (4):239-245.

4. Tewari A, Sood N, Vegad MM, Mehta DC. "Epidemiological and microbiological profile of infective keratitis in Ahmedabad". Indian J Ophthalmol 2012; 60:267-72.

5. Suman Saha, Debdulal Banerjee, Archana Khetan, "Epidemiological profile of fungal keratitis in urban population of West Bengal, India". Oman Journal of Ophthalmology, 2009; 2(3):114-118. 
6. M Jayahar Bharathi, R Ramakrishnan, Samala Vasu, "Epidemiological Characteristics and Laboratory Diagnosis of Fungal Keratitis. A Three-year Study". Indian Journal of Ophthalmolgy,( 2003) 51(3)15-21.

7. Usha Gopinathan, Savitri Sharma, Prashant Garg, Gullapalli N Rao. "Review of epidemiological features, microbiological diagnosis and treatment outcome of microbial keratitis: Experience of over a decade". Indian J Ophthalmol: 2009;57:273-279 